\title{
Modelling vortex-induced vibration with driven oscillation
}

J. S. Leontini* M. C. Thompson* $\quad$ K. Hourigan*

(received 19 October 2004, revised 8 March 2005)

\begin{abstract}
Two-dimensional simulations of flow past an elastically mounted cylinder, and flow past an externally driven oscillating cylinder were performed at a Reynolds number of 200. The results of both are compared to see if the driven oscillation could model the coupled fluidstructure flow of the elastically mounted cylinder. The driven system could model the elastically mounted system, but was very sensitive to input parameters. We argue that this sensitivity could cause experimental discrepancies between the two systems.
\end{abstract}

${ }^{*}$ Dept. of Mechanical Engineering, Monash University, Clayton, Australia. mailto: justin. leontini@eng. monash. edu . au

See http://anziamj.austms.org.au/V46/CTAC2004/Leon for this article, (C) Austral. Mathematical Soc. 2005. Published May 15, 2005. ISSN 1446-8735 
TABLE 1: nomenclature.

$A^{*} \quad$ Amplitude ratio, $y / D$

$C_{L} \quad$ Lift coefficient, $F_{L} /\left(0.5 \rho \mathrm{v}^{2} D\right)$

$C_{E} \quad$ Energy transfer coefficient, $\int_{T} C_{L} \mathrm{v} \mathrm{d} t$

$D$ Cylinder diameter

$F_{L} \quad$ Lift force / unit length

$f$ Oscillation frequency

$f^{*} \quad$ Normalised oscillation frequency, $f D / U$

$f_{N} \quad$ Natural structural frequency

$f_{N}^{*} \quad$ Normalised natural frequency, $f_{N} D / U$

$f_{\mathrm{St}} \quad$ Vortex-shedding frequency from a stationary cylinder

$m^{*} \quad$ Mass ratio, $m_{\mathrm{CYL}} /\left(\frac{\pi}{4} D^{2} \rho L\right)$

Re Reynolds number, $U D / \nu$

St Strouhal number, $f_{\mathrm{St}} D / U$

$U$ Free-stream velocity

v Transverse cylinder velocity

y Transverse cylinder displacement

$\zeta$ Damping ratio, $c / c_{\mathrm{CRIT}}$

\section{Contents}

1 Introduction

C367

2 Computational method

C368

3 Results

C370

3.1 Vortex-induced vibration . . . . . . . . . . . . . C C370

3.2 Synchronisation during driven oscillation . . . . . . . . C C371

3.3 Energy transfer during driven oscillation . . . . . . . . . C373

4 Conclusions

C376

References 


\section{Introduction}

Vortex-induced vibration (VIV) of bluff structures can occur whenever a bluff structure is immersed in a fluid stream. If the frequency of this vibration is close to a natural frequency of the bluff structure, large scale oscillations occur, ultimately resulting in the failure of the structure.

In an attempt to study the fundamental aspects of VIV, simplified experimental models are used. The most common of these is an elastically mounted cylinder constrained to oscillate across the flow [5, 8, 6, e.g.]. While simplified, this model is still subject to the complexity of coupled fluid-structure interaction.

As the steady-state oscillations of the elastically mounted cylinder often appear sinusoidal, it has often been hypothesised that the fluid and structural systems can be decoupled. The oscillation is then externally driven, rather than responding to the flow. The transverse driven oscillating cylinder has been studied by many $[1,13,4$, e.g.].

The results from driven oscillation experiments, in comparison to VIV experiments, are mixed. An accurate comparison can be made between the wakes generated, and the general flow topology. However, the energy transfer coefficient $C_{E}$ during driven cylinder experiments has been shown to be negative whereas it is positive during VIV at similar flow conditions. During experiments, the sign of $C_{E}$ is inferred from the sign of the phase angle between the lift force on the cylinder, and the cylinder displacement [2]. The discrepancy means that the driven oscillation experiments predict no vibration, when in fact VIV occurs at its largest amplitudes.

Some attempts have been made at directly predicting VIV from driven oscillation results. Sarpkaya [9] had only limited success, and prescribed a limiting amplitude during VIV that has since been exceeded [7]. Subsequently Staubli [10] only found agreement between prediction and experiments over a small range of oscillation frequencies. The more recent direct comparison 
of [3] showed these discrepancies quite clearly. This discrepancy recently led Williamson \& Govardhan [12] to ponder whether it was at all possible to model VIV with pure-tone driven oscillation.

Here we hypothesise that it is possible to model VIV with driven oscillation if the frequency and amplitude of oscillation are matched closely $(<1 \%$ error $)$. The results we present were obtained at a Reynolds number $\operatorname{Re}=200$, using two-dimensional simulation. While this flow is inherently two-dimensional, and the Re used during experiments render the flow threedimensional, the results shown seem promising for the modelling of VIV with driven oscillation.

\section{Computational method}

A spectral-element method was employed to solve the two-dimensional incompressible Navier-Stokes equations. A fuller description of this method is given in [11]. An accelerated frame of reference was employed that was fixed to the cylinder, to allow the cylinder to oscillate across the stream in both the VIV and driven oscillation simulations. A 508 macro-element mesh with overall dimensions of $30 \mathrm{D}$ downstream, and $15 \mathrm{D}$ upstream and to either side, was used. This mesh is shown in Figure 1.

Eighth-order tensor product Lagrange polynomials, associated with Gauss-Legendre-Lobatto interpolation points, were employed over each element. This rendered the scheme used as an $h-p$ method. This means that the macro spatial discretisation $h$ could be changed separately to the order $p$ of the interpolating polynomials.

A grid resolution study was performed by incrementing the order $p$ of the polynomials, and performing simulations of flow past a fixed cylinder. The parameter compared between resolutions was the Strouhal number St. The value of $\mathrm{St}=0.198$ was found to vary by $<1 \%$ for $p>6$. A polynomial 


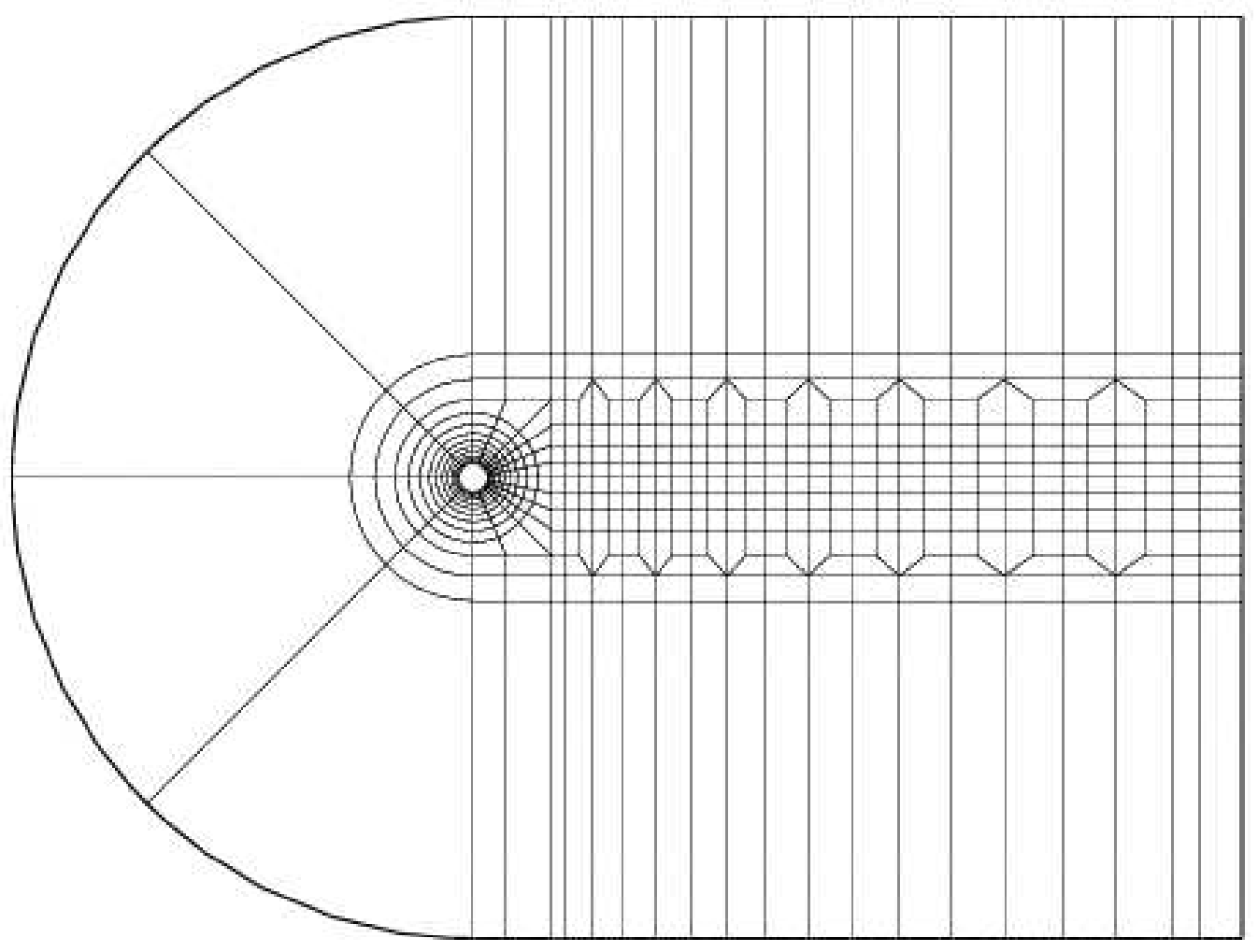

FiguRE 1: The mesh used for both the VIV and driven oscillation simulations. 
order $p=8$ was therefore employed throughout this study.

A second order time-splitting scheme was used for both the VIV and driven oscillation cases. The time integration for the VIV simulations employed a semi-implicit scheme, with an Adams-Bashforth 3rd-order routine used to obtain the first guess used during the internal iteration. This was required due to the coupling of the flow and cylinder movement. Two time steps, $\delta \tau=$ 0.005 and $\delta \tau=0.01$ were tested with the semi-implicit scheme. It was found that at $U^{*}=5, f^{*}$ and $A^{*}$ differed by $<1 \%$. Therefore, a time step of $\delta \tau=$ 0.01 was employed throughout this study with confidence that this time step produced physically meaningful results. The driven oscillation simulations used an explicit integration scheme, as the cylinder position was prescribed externally. Interestingly, while each time step of the explicit scheme used less CPU time than the semi-implicit scheme, the time step employed was an order of 10 smaller, resulting in more CPU time overall.

\section{Results}

\subsection{Vortex-induced vibration}

To allow a comparison between vortex-induced vibration (VIV) and driven oscillations, a set of VIV results were obtained. For all of these simulations, a mass ratio $m^{*}=10$, where $m^{*}$ is the ratio between the mass of the cylinder structure and the mass of displaced fluid. A damping coefficient was used that yielded a damping ratio $\zeta=0.01$, resulting in a combined mass-damping parameter $m^{*} \zeta=0.1$.

To vary the oscillation frequency, the natural frequency of the system was varied by altering the value of the spring constant $k$. Three distinct regions of amplitude response were observed, with the highest magnitude response occurring at natural frequencies close or equal to the shedding frequency of 
a stationary cylinder, $f_{\mathrm{St}}$. At these natural frequencies, the shedding frequency of the wake was observed to synchronise with the natural frequency of the structure. Therefore, this response regime was labeled the synchronisation region. Outside this region, shedding was at approximately the same frequency as the shedding from a fixed cylinder, $f_{\mathrm{St}}$.

This synchronisation region can be clearly identified in Figure 2a and $2 \mathrm{~b}$. Figure 2a shows $A^{*}$ plotted against the normalised response frequency $f^{*}$. This plot clearly shows the sudden jump to larger amplitudes with synchronisation, and the sudden decrease in amplitude of oscillation with the loss of synchronisation. Figure $2 \mathrm{~b}$ shows the ratio of response frequency $f$ to the natural frequency $f_{N}$, plotted against the normalised response frequency $f^{*}$. This plot shows the sudden synchronisation of the wake to the natural frequency, as the natural frequency approaches the wake shedding frequency of the stationary cylinder, $f_{\mathrm{St}}$.

\subsection{Synchronisation during driven oscillation}

Presented in Figure 3 are the synchronisation boundaries for the driven cylinder case, in the $A^{*}$ versus $f^{*}$ plane. Also plotted are those points from VIV cases where synchronisation occurred. See that almost all of the VIV cases that synchronised fall inside the driven oscillation synchronisation boundaries. This indicates that during synchronisation, the cylinder oscillation has control of the wake, and overrides the 'natural' Von Karmen instability that causes the wake to oscillate in the stationary cylinder case. Also, as only pure-tone driven oscillation was used during this investigation, it seems that it is this fundamental frequency that has the major effect on synchronisation.

Whereas the lower frequency synchronisation boundary presented in Figure 3 is well defined, the upper boundary is not and requires further work to clearly define. This boundary is difficult to define due to the high sensitivity of the system to input frequency. For a given amplitude, varying 
(a)

(b)
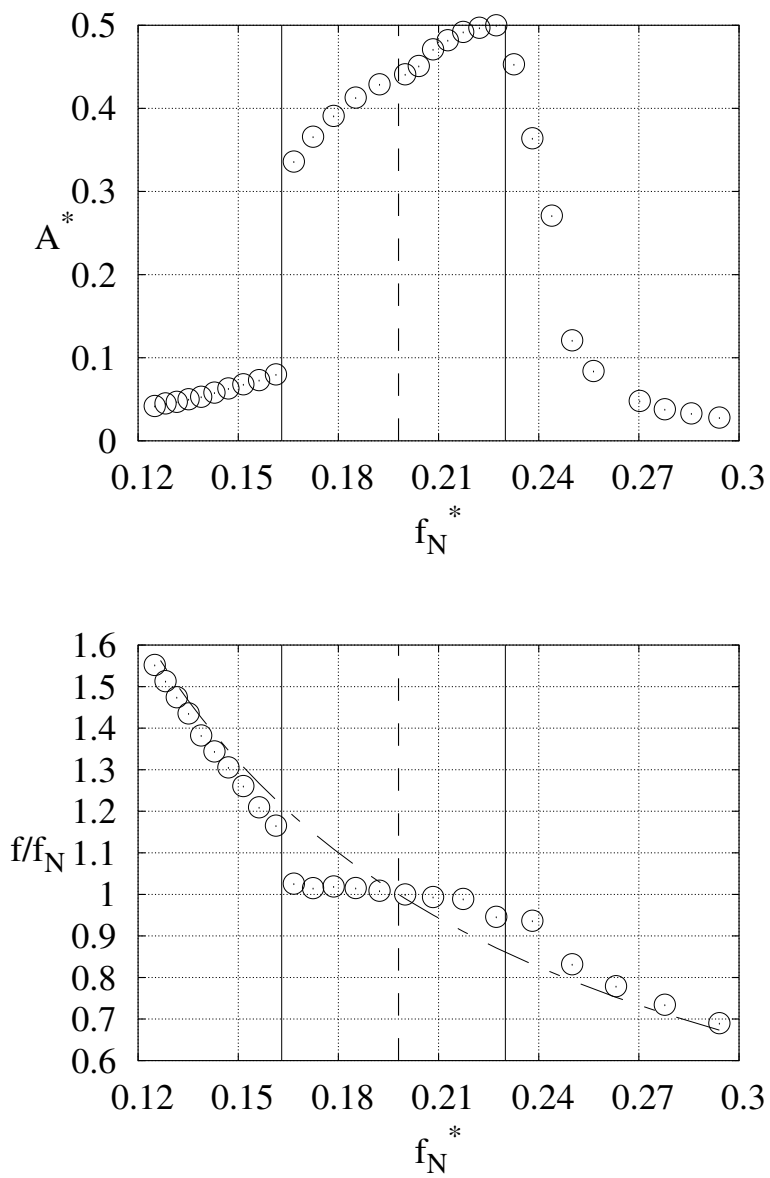

Figure 2: (a) $A^{*}$ versus normalised natural frequency. The largest amplitudes occur between $f_{N}^{*}=0.16$ and $f_{N}^{*}=0.22$, bracketing the shedding frequency from a stationary cylinder, $f_{\mathrm{St}}(---)$. (b) The ratio of response frequency $f$ to natural frequency $f_{N}$, versus normalised natural frequency $f_{N}^{*}$. The dash-dot line (- - - ) is $f_{\mathrm{St}} / f_{N}^{*}$, showing that outside the synchronisation region, the vortices shed at approximately the fixed cylinder frequency $f_{\text {St }}$. 


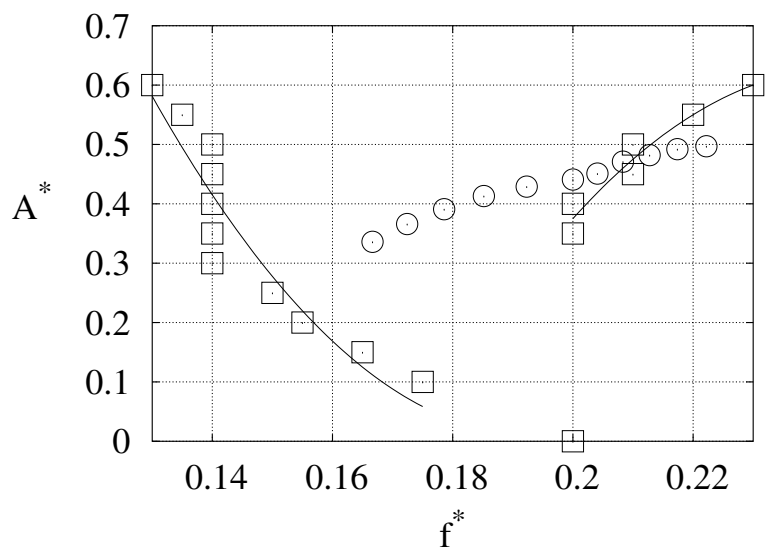

FiguRE 3: Synchronisation boundaries for the driven oscillation case. $\square$ represents data points used for synchronisation boundary. - represents line of best fit for synchronisation boundary. o represents VIV synchronised cases.

the normalised oscillation frequency by as little as $10^{-3}$ could change the magnitude of the lift force on the cylinder by as much as $25 \%$. It could also result in synchronisation taking up to 5 times as long, or completely inhibit synchronisation.

\subsection{Energy transfer during driven oscillation}

For driven oscillation to be a useful model of VIV, it needs to exhibit similar energy transfer characteristics to the VIV case. Energy transfer is quantified as the work done on the cylinder by the fluid over one cycle of oscillation. This quantity is normalised to return an energy transfer coefficient $C_{E}$.

During steady-state, synchronised VIV, the overall energy transfer to the mechanical system must be zero. Therefore, if any damping is present in the system, it is expected that the work done on the cylinder by the fluid will be equal to the work done by mechanical damping. Hence, $C_{E}$ during VIV 


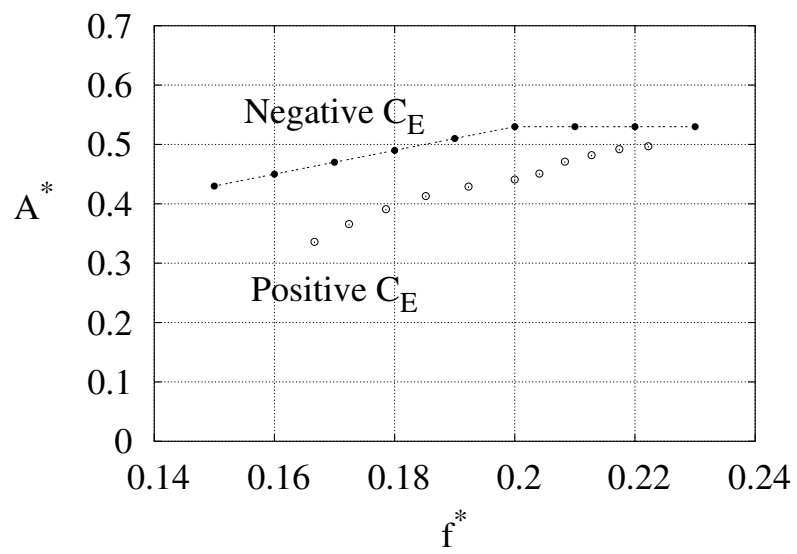

FiguRE 4: Energy transfer directions for the driven oscillating cylinder. - • represents $C_{E}=0$. o represents VIV amplitudes of oscillation.

retains the same definition as during driven oscillation. For driven oscillation to model VIV, it must return a positive value of $C_{E}$ in regions of the $A^{*}-f^{*}$ plane where VIV occurs.

Figure 4 shows points of $C_{E}=0$, effectively dividing the $A^{*}-f^{*}$ plane into $C_{E}>0$ and $C_{E}<0$ zones. It shows an upper limit on $A^{*}$ for $C_{E}>$ 0 , indicating a limit on the amplitude possible during VIV, if the driven oscillation is modelling VIV accurately.

Also plotted are the amplitudes of VIV cases, and all of these fall in the $C_{E}>0$ region. This result is encouraging, and indicates that at the Reynolds number tested, the driven oscillation using a pure-tone oscillation is capturing most of the characteristics of VIV. It also shows that the VIV cases occur close to the transition from positive to negative $C_{E}$. The small positive difference can be attributed to the energy required by the system to account for energy lost through damping.

The proximity of the VIV cases to the boundary between positive and negative $C_{E}$ again highlights the importance of matching the input frequency 


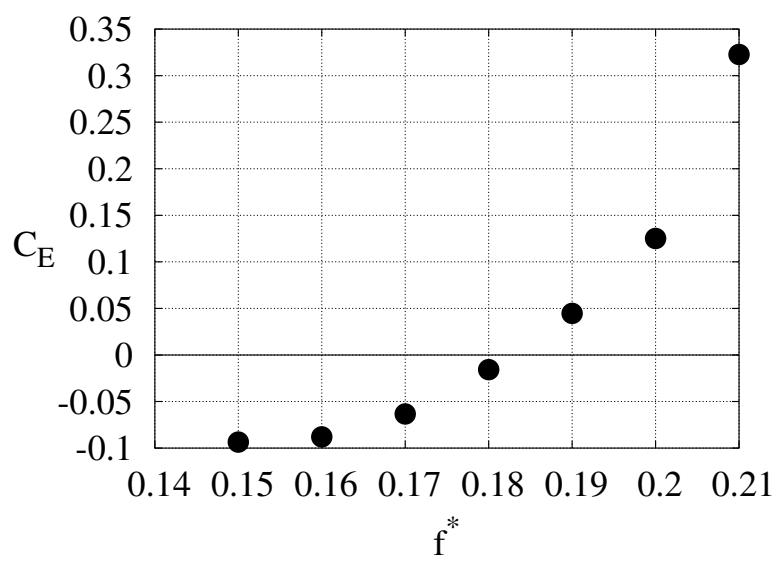

Figure 5: $C_{E}$ against $f^{*}, A^{*}=0.5$.

closely, as high gradients of $C_{E}$ are observed in this area of the parameter space. This is illustrated by plotting the values of $C_{E}$ versus $f^{*}$, for a given amplitude, as done for an amplitude $A^{*}=0.5$, shown in Figure 5. It is shown that as soon as $C_{E}$ becomes positive, it quickly increases with increasing frequency $f^{*}$. This highlights the high sensitivity of the driven system. While a good match between the VIV and driven oscillation can be obtained if the frequency and amplitude of oscillation are matched, this matching must be very accurate (discrepancies of $<1 \%$ ), otherwise significant differences in behaviour will be observed.

A similar sensitivity is observed in the value of $A^{*}$. Changes in the magnitude of $A^{*}$ as small as $1 \%$ can result in changes in the lift force on the cylinder of the order of $25 \%$, resulting in similarly significant changes in the value of $C_{E}$. This sensitivity is highlighted in Figure 6. $C_{E}$ is plotted against $A^{*}$ for a fixed normalised oscillation frequency $f^{*}=0.20 . C_{E}$ is shown to quickly vary as the amplitude of oscillation $A^{*}$ approaches the transition from positive to negative $C_{E}$. 


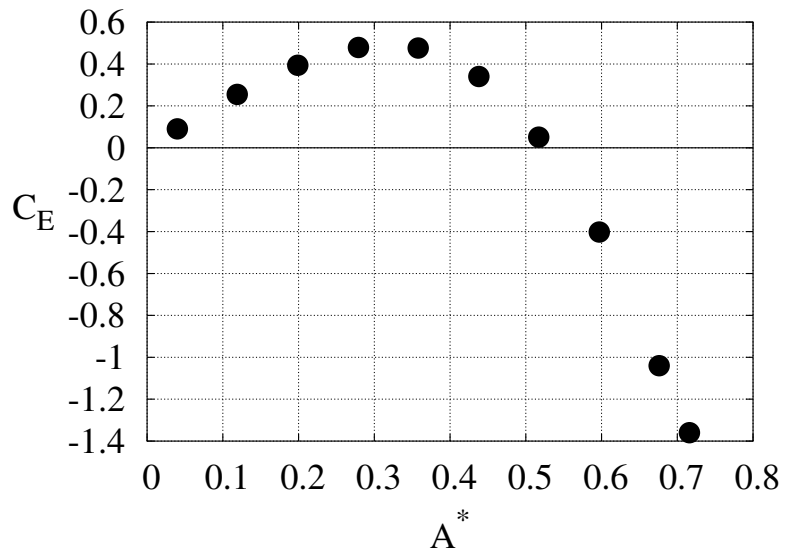

Figure 6: $C_{E}$ against $A^{*}, f^{*}=0.2$.

\section{Conclusions}

Experimental work has been unable to capture all of the characteristics of vortex-induced vibration, especially the energy transfer characteristics. However, this numerical study has made good progress, showing that VIV occurs where it is expected, and the oscillations it causes are well predicted by driven oscillation simulations.

This numerical study has shown that the driven oscillating system is highly sensitive to the amplitude of oscillation $A^{*}$, and the oscillation frequency $f^{*}$. Changes of the order of $1 \%$ in either can change the magnitude of the energy transfer coefficient $C_{E}$ by the order of $25 \%$. In some critical instances, this can also result in the sign of $C_{E}$ reversing.

While the Reynolds number is low compared to experiments, the discovery of this high sensitivity could provide an explanation as to why driven oscillation experiments predict VIV should not occur when it actually occurs at its largest amplitudes. It remains to be seen whether three-dimensional simulations at higher Reynolds number can improve the predictions made. 
Acknowledgments: Primary support for this research program was provided by the Monash University Engineering research committee. Mr. Leontini also acknowledges support from the Department of Mechanical Engineering, Monash University, through a Departmental Postgraduate Scholarship.

\section{References}

[1] R. E. D. Bishop and A. Y. Hassan. The lift and drag forces on a circular cylinder oscillating in a flowing fluid. Proceedings of the Royal Society of London, series A, 277, n1368:51-75, 1964. C367

[2] J. Carberry. Wake states of a submerged oscillating cylinder and of a cylinder beneath a free surface. PhD thesis, Monash University, 2001. C367

[3] J. Carberry, J. Sheridan, and D. Rockwell. A comparison of forced and free oscillating cylinders. In Proceedings of the 14th Australasian Fluid Mechanics Conference, pages 701-704, 2001. C368

[4] J Carberry, J. Sheridan, and D. Rockwell. Forces and wake modes of an oscillating cylinder. J. Fluids \& Structures, 15:523-532, 2001. C367

[5] C. C. Feng. The measurement of vortex-induced effects in flow past a stationary and oscillating circular and d-section cylinders. Master's thesis, University of British Columbia, 1968. C367

[6] R. Govardhan and C. H. K. Williamson. Modes of vortex formation and frequency response of a freely vibrating cylinder. J. Fluid Mech., 420:85-130, 2000. C367

[7] A. Khalak and C. H. K. Williamson. Dynamics of a hydroelastic cylinder with very low mass and damping. J. Fluids \& Structures, 10:455-472, 1996. C367 
[8] A. Khalak and C. H. K. Williamson. Motions, forces and mode transitions in vortex-induced vibrations at low mass-damping. J. Fluids \& Structures, 13:813-851, 1999. C367

[9] T. Sarpkaya. Fluid forces on oscillating cylinders. Journal of the waterways, port, coastal and ocean division of ASCE, 104:275-290, 1978. C367

[10] T. Staubli. Calculation of the vibration of an elastically mounted cylinder using experimental data from forced oscillation. J. Fluids Eng., 105:225-229, 1983. C367

[11] M. C. Thompson, K. Hourigan, and J. Sheridan. Three-dimensional instabilities in the wake of a circular cylinder. Experimental Thermal and Fluid Science, 12:190-196, 1996. C368

[12] C. H. K. Williamson and R. Govardhan. Vortex-induced vibrations. Annu. Rev. Fluid Mech., 36:413-455, 2004. C368

[13] C. H. K. Williamson and A. Roshko. Vortex formation in the wake of an oscillating cylinder. J. Fluids \& Structures, 2:355-381, 1988. C367 